\title{
Temporally Smoothed Wavelet Coherence for Multivariate Point-Processes and Neuron-Firing
}

\author{
Alex J. Gibberd \\ Department of Mathematics and Statistics \\ Lancaster University \\ Lancaster, UK \\ Email: a.gibberd@lancaster.ac.uk
}

\author{
Edward A. K. Cohen \\ Department of Mathematics \\ Imperial College London \\ London, UK \\ Email: e.cohen@imperial.ac.uk
}

\begin{abstract}
In neuroscience, it is of key importance to assess how neurons interact with each other as evidenced via their firing patterns and rates. We here introduce a method of smoothing the wavelet periodogram (scalogram) in order to reduce variance in spectral estimates and allow analysis of time-varying dependency between neurons at different scale levels. Previously such smoothing methods have only received analysis in the setting of regular real-valued (Gaussian) timeseries. However, in the context of neuron-firing, observations may be modelled as a point-process which when binned, or aggregated, gives rise to an integer-valued time-series. In this paper we propose an analytical asymptotic distribution for the smoothed wavelet spectra, and then contrast this, via synthetic experiments, with the finite sample behaviour of the spectral estimator. We generally find good alignment with the asymptotic distribution, however, this may break down if the level of smoothing, or the scale under analysis is very small. To conclude, we demonstrate how the spectral estimator can be used to characterize real neuron-firing dependency, and how such relationships vary over time and scale.
\end{abstract}

\section{INTRODUCTION}

It is often considered to treat neuronal firing patterns as a multivariate point process $\left\{N(t) \equiv\left(N_{1}(t), \ldots, N_{p}(t)\right)^{\prime} \mid t \in\right.$ $(0, T]\}$, where $N_{i}(t)$ counts the number of events of class $i$ for $i=1, \ldots, p$ data-streams up until a point $t$ [Brillinger et al., 1976, Halliday and Rosenberg, 1999, Jarvis and Mitra, 2001]. A topic of prevailing interest over the years [Brillinger, 1992, Ventura et al., 2005, Tang et al., 2015] has been to investigate how neurons, or groups thereof, interact under different environmental situations or subject to stimulation. One very popular measure of association for neurological time-series is given by the Fourier coherence [Jarvis and Mitra, 2001, Bartlett, 1963], and traditionally defined by the correlation of Fourier coefficients, or in relation to the cross-spectra $S_{i j}(f)$ according to

$$
\gamma_{i j}^{2}(f)=\left|S_{i j}(f)\right|^{2} / S_{i i}(f) S_{j j}(f),
$$

for the $i$ and $j$ th stream at frequency $\omega$. However, in many, if not most settings, one expects that the dependency properties of firing patterns may change over time, and hence the data must be assumed to be generated from a non-stationary process. Unfortunately, since the support of the Fourier series is not temporally localised, traditional Fourier based methods fail to characterise these important time-evolving properties.

\section{Wavelet Coherence}

To tackle this lack of temporal sensitivity, there has been an interest in using localised short-time Fourier transform (STFT) and continuous wavelet transform (CWT) methods [c.f. Halliday and Rosenberg, 1999, Hramov et al., 2015] to assess the temporally-localised spectral properties of neuronal activity. Such methods are well developed in the traditional time-series setting [c.f. Zhan et al., 2006, Cohen and Walden, 2010, Bigot et al., 2011], however, are still relatively unexplored in the point-process setting. To our knowledge, the preliminary work of Cohen [2014] represents the first study of the CWT being applied directly to pointprocess event data. In this work, by considering a binned point-process, we take an approach in-between the two extremes of regular (continuous) time-series analysis, and that of studying the event data itself.

Specifically, let $X_{i}[k] \equiv N_{i}(k \Delta)-N_{i}((k-1) \Delta)$ be the count of events in an interval $\mathcal{S}_{k}:=[(k-1) \Delta, k \Delta)$, $k \in \mathbb{Z}$, for stream $i \in\{1, \ldots, p\}$ with binning width $\Delta$. Further, we may define the multivariate count process $X[k] \equiv\left(X_{1}[k], \ldots, X_{p}[k]\right)^{\prime}=N(k \Delta)-N((k-1) \Delta)$. Now, consider the CWT of a continuous time function $g(t)$, where the wavelet coefficient at shift $b^{\prime} \in \mathbb{R}$ and scale $a^{\prime} \in \mathbb{R}$ is given as

$$
W\left(a^{\prime}, b^{\prime} ; g, \psi\right):=\frac{1}{\sqrt{\left|a^{\prime}\right|}} \int_{-\infty}^{\infty} g(t) \psi^{*}\left(\frac{t-b^{\prime}}{a^{\prime}}\right) d t .
$$

A key quantity of interest in this paper is the set of empirical wavelet coefficients of the process $\bar{X}[k]:=X[k]-E\{X[k]\}$, an approximation of the stochastic process $\{Z(t)\}$ defined as $Z(t):=d N(t) / d t-\lambda$, where $\lambda \equiv E\{d N(t)\} / d t$.

The CWT of $\{Z(t)\}$ on the finite length time interval $t \in(0, T]$ can be approximated as a discrete convolution in the Fourier domain

$W\left(a^{\prime}, b^{\prime} ; Z, \psi\right) \simeq \frac{\left|a^{\prime}\right|^{1 / 2}}{T \Delta} \sum_{q=-N / 2}^{N / 2-1} \tilde{Z}_{T}\left(f_{q}\right) \tilde{\psi}^{*}\left(a^{\prime} f_{q}\right) e^{i 2 \pi b^{\prime} f_{q}}$

where $\tilde{Z}_{T}$ indicates the Fourier transforms of $\{Z(t)\}$ on $(0, T], \tilde{\psi}$ is the Fourier transform of $\psi$, and $f_{q}=q / N$ for $q=1, \ldots, N$ with $T=N \Delta$. The approximation becomes accurate as $T \rightarrow \infty$ as long as $\left|\tilde{Z}_{T}(f)\right|=0$ for $|f|>$ 
$f_{\mathrm{Nyq}}=1 / 2 \Delta$. Through the further approximation $\{\bar{X}[k]\}$ of $\{Z(t)\}$, we can consider the discrete Fourier transform of the binned sequence $\tilde{\bar{X}}[q]=\Delta \sum_{k=0}^{N-1} \bar{X}[k] e^{-i 2 \pi k q / N}$ in the place of $\tilde{Z}_{T}\left(f_{q}\right)$ to obtain

$$
V(a \Delta, b \Delta ; X, \psi):=\frac{|a|^{1 / 2}}{\Delta^{1 / 2} N} \sum_{q=0}^{N-1} \tilde{\tilde{X}}[q] \tilde{\psi}_{a}[q] e^{i 2 \pi b q / N},
$$

where $a, b \in \mathbb{Z}$ are now integer valued, and

$$
\tilde{\psi}_{a}^{*}[q]=\left\{\begin{array}{ll}
\tilde{\psi}^{*}(a q / N) & q=0, \ldots,(N / 2)-1 \\
\tilde{\psi}^{*}(a(q-N) / N) & q=(N / 2), \ldots, N-1
\end{array} .\right.
$$

We note, as the number of bins in the support of the wavelet transform goes to infinity, then $V(a \Delta, b \Delta ; X, \psi)$ becomes an increasingly accurate approximation $W(a \Delta, b \Delta ; Z, \psi)$.

To estimate quantities such as the wavelet spectra, or the wavelet coherence (analogous to Eq. 1), we consider estimates based on the wavelet periodogram (or scalogram), this is defined as the outer-product of the empirical (approximate) wavelet coefficients

$$
I^{(X)}[a, b]:=V(a \Delta, b \Delta ; X, \psi) V^{H}(a \Delta, b \Delta ; X, \psi) .
$$

Furthermore, in order to reduce variance in the estimator, we will smooth this quantity by averaging over $M$ time-points in the region of $b$, i.e.

$$
\hat{S}^{(X)}[a, b]:=M^{-1} \sum_{k=b-M / 2}^{b+M / 2} I^{(X)}[a, k] .
$$

\section{Contribution}

In the previous work of Cohen and Walden [2010] it was demonstrated that when using the Morlet wavelet, asymptotically, as $a \rightarrow \infty$ and $T \rightarrow \infty$ we have $\hat{S}^{(Y)}[a, b] \simeq$ $\mathcal{W}_{C}\left(0, S^{(Y)}(1 / a \Delta)\right)$ where one discretely samples a realvalued second-order stationary Gaussian process $\{Y(t)\}$ with Fourier spectra $S^{(Y)}(f)$. In the case of a point-process, the binned counting process $\{X[k]\}$ is integer valued, and necessarily does not provide full coverage of the real line. In this paper we propose that the asymptotic distribution for $\hat{S}^{(X)}[a, b]$ is similar to that given in Theorem 1, Cohen and Walden [2010], and that this also holds in the binned pointprocess setting, we summarise this proposition below. Note: all the analysis from here will be formed on the basis that $\psi$ is given by the Morlet wavelet, this is simply a Gaussian tapered exponential basis.

Proposition 1 (Asymptotic Distribution of Smoothed Periodogram). Let $\{X[k]\}$ be a multivariate second-orderstationary count process derived from a binned $(\Delta)$ detrended point-process $\{Z(t)\}$ with true second order Fourier spectra $S^{(Z)}(f) \in \mathbb{C}^{p \times p}$. Under suitable mixing conditions (limited range dependency, as in Assumption 2.1, 2.2 Brillinger, 1972) then the smoothed periodogram for the Morlet wavelet is complex Wishart distributed

$$
\hat{S}^{(X)}[a, b] \triangleq \mathcal{W}_{C}\left\{K^{-1} S^{(Z)}(1 / a \Delta), K\right\},
$$

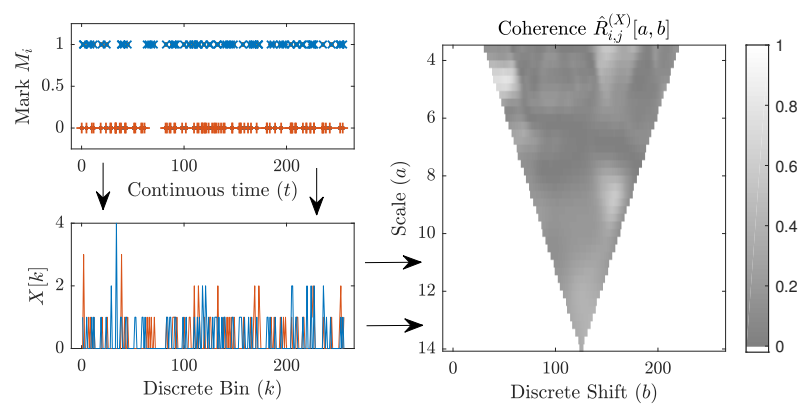

Figure 1. An example of the processing pipeline, from continuous time point-process, to wavelet coherence is demonstrated in Figure 1. The shaded area in the coherence plot represents values in the supported set $(a, b) \in \mathcal{C}_{T}$ for $\kappa=12, \Delta=1$ and $T=256$.

where $K$ is the degrees of freedom and can be calculated according to the method of Walden 2000, Cohen and Walden 2010.

For the purposes of this paper, we do not consider the proof of the above, rather, we consider what the sampling distribution looks like in practice, i.e. in more realistic finite data settings, and particularly for fine scale levels, or small binning intervals. We contrast these results with Proposition 1 to assess how reasonable it is to use the asymptotically derived distribution for inference purposes. We assess the practicality of this distribution through synthetic experiments with independent Poisson processes where we know the true spectral structure. Additionally, we apply the smoothed discrete time wavelet estimator (3) to real binned neuronfiring data to examine the ability of the method to detect and characterise coherent signalling between neurons.

\section{The Empirical Distribution of the SMOOTHED WAVELET SPECTRA}

In this section, we assess the distributional properties of the wavelet spectra under a range of binning, smoothing, and firing rate parameters. For simplicity, we here utilise independent Poisson point-processes as the generative model, thus the true spectra for the process is flat, i.e. $S^{(Z)}(f)=\lambda$ for all $f$. Rather than consider directly the distribution, for the sake of interpretation we assess the wavelet coherence, analogously to 1 , we define

$$
\hat{R}_{i j}^{(X)}[a, b]=\left|\hat{S}_{i j}^{(X)}[a, b]\right|^{2} / \hat{S}_{i i}^{(X)}[a, b] \hat{S}_{j j}^{(X)}[a, b],
$$

and let $R_{i j}^{(X)}[a, b]$ denote the true coherence.

Corollary 2. It follows from Prop. 1 that the estimator is asymptotically (under the same conditions) Goodman [Goodman, 1963] distributed, i.e. $\hat{R}_{i j}^{(X)}[a, b] \triangleq$ $\operatorname{Goodman}\left(R_{i j}^{(X)}[a, b], K\right)$.

Of course, the suitability of Cor. 2 depends on the suitability of Prop. 1. In the experiments below we assess the coverage properties of the proposed asymptotic distribution (Cor. 2) in the setting where the true coherence is zero, i.e. $R_{i j}^{(X)}[a, b]=0$ for all $[a, b] \in \mathcal{C}_{T}$ and $\mathcal{C}_{T} \subset \mathbb{Z}_{+}^{2}$ is a 

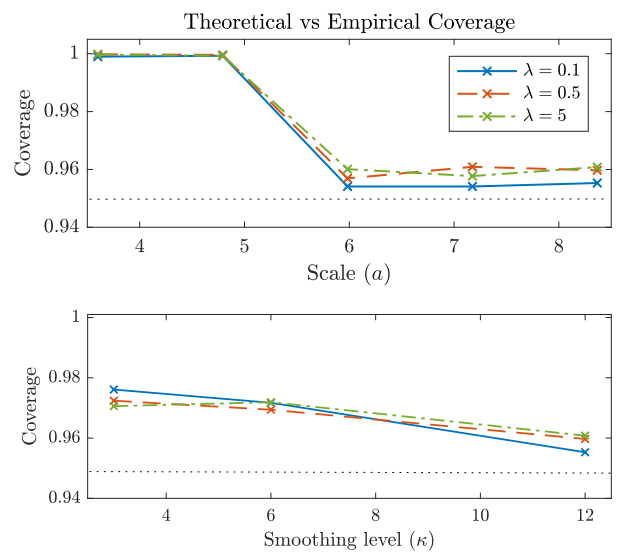

Figure 2. Empirical proportion of coherence estimates that are smaller than the 95th percentile of the asymptotic distribution for: Top) different scales $a$ with fixed smoothing parameter $\kappa=12, K \approx 10$; Bottom) different smoothing levels $\kappa$ at the maximum relative scale $a / a_{\max }=1$. All experiments performed with fixed bin size $\Delta=1$ and $N_{\exp }=10,000$ repetitions.

set of allowable sampling points in the discrete scale-time plane. Note: the boundary of the set is derived by the level of smoothing required $M$, and the approximate support of the wavelet $\psi_{\text {Morlet }}(t)$, see the example coherence estimate in Figure 1, and Cohen and Walden [2010] Section 3 for details.

\section{Experimental setup}

For simplicity, we consider the bivariate $p=2$ case with two independent Poisson point-processes, furthermore, we assume that $\lambda_{1}=\lambda_{2}$. From this, we construct a bivariate discrete time process $\left\{\left(X_{1}[k], X_{2}[k]\right)^{\prime}\right\}$ for $k=1, \ldots, N$ via binning over intervals of size $\Delta$. In all experiments we keep $T:=N \Delta=256$, however, we vary $\lambda$ and $M=\kappa a$ the smoothing width to assess how coverage varies under different levels of smoothing. To calculate the degrees of freedom $K$ we use the method suggested in Walden [2000], Cohen and Walden [2010] based on a multi-taper reformulation of Eq. 3 .

The results of this experiment are presented in Figs. 2, 3 , which respectively present the empirical proportion of coherence estimates falling below the 95th percentile of the asymptotic distribution, and the overall distributional alignment as assessed via QQ-plot. One immediate thing to notice is that the coverage of the Goodman derived interval (nominally set at $\alpha=0.05$ ) appears to be conservative in that the number of estimates below this level exceeds the nominal rate. The Goodman derived bounds appear conservative for a range of both scale levels and smoothing levels. However, as seen in Fig. 2, it appears that the nominal coverage is approached as $\kappa$ and/or $a$ increase. Interestingly, these results seem robust to the process rate parameter, in fact, as far as these experiments are concerned the asymptotic distribution appears to be more appropriate when using a smaller $\lambda$ (compare the bottom figures in Fig. 3). The QQ-plot analysis augments that of the coverage (which may be sensitive to
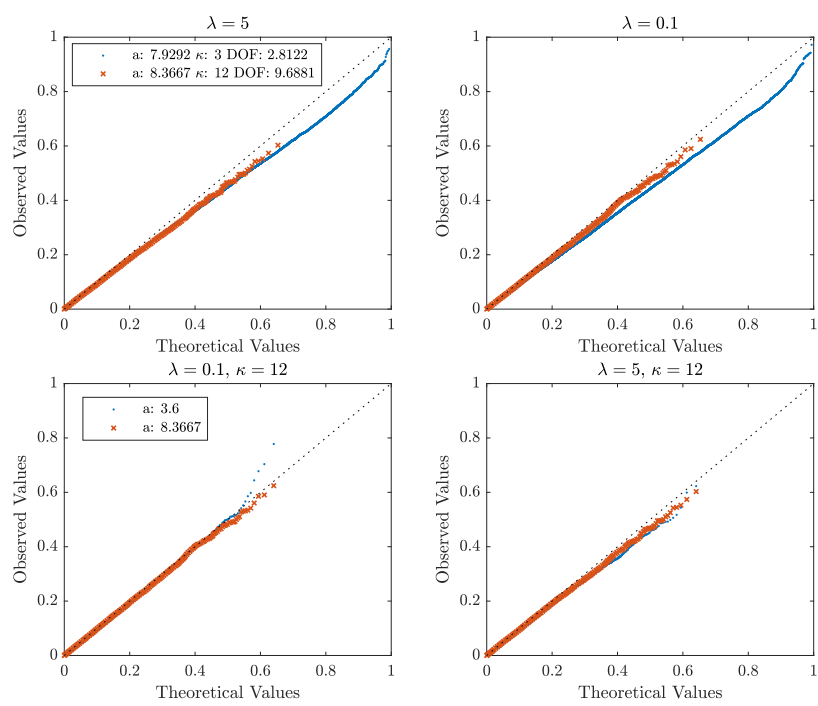

Figure 3. QQ-plots comparing the theoretical and empirical distribution for the temporally smoothed wavelet coherence. Top: We compare different levels of smoothing $\kappa$, however, keep the scales similar $a \approx 8$ giving rise to different degrees of freedom. Bottom: A comparison of the distributions under different firing rates $\lambda$, but the same smoothing parameter. In this case, the degrees of freedom are simillar for both plotted values.

tail values) and provides a broader picture of alignment between the theoretical and empirical distributions. For large smoothing values $\kappa=12$ and greater, the alignment seems very good between theoretical and observed quantiles, this is not always the case when using smaller smoothing windows (i.e. smaller values of $\kappa$ ). For instance, the top plots in Fig. 3 indicate that the observed values systematically lie below those expected. Not only does this explain some of the behaviour in the coverage analysis, it also suggests that we should be careful to use the theoretical Goodman distribution in low $\kappa$ settings.

\section{WAVElet COHERENCE OF NEURONAL Firing}

In this section we apply the smoothed wavelet coherence estimator (3) to real neuron-firing data. In this case, we study a subset of firing data relating to the lateral geniculate nucleus (LGN) as presented in the study of Tang et al. [2015]. In that paper, the authors are primarily interested in characterising the marginal firing properties of neurons in order to understand how neurons encode and transfer visual signals. In our case, we will consider second-order neuronneuron dependency properties, and how these vary across scale via the smoothed wavelet spectra. We here consider again the simple case of $p=2$ neurons to illustrate the method. A full neuroscientific analysis of firing patterns is beyond the scope of this paper, and as such we defer this to future work.

Based on the results of Section 2, we here decide to analyse only a subset of firing recordings which have a relatively large number of events in each stream. A set of event traces for the pair of cells presented here is given in Figure 4. The recordings used start at the time of a visual intervention, and end approximately $T=2 \mathrm{~ms}$ later. 

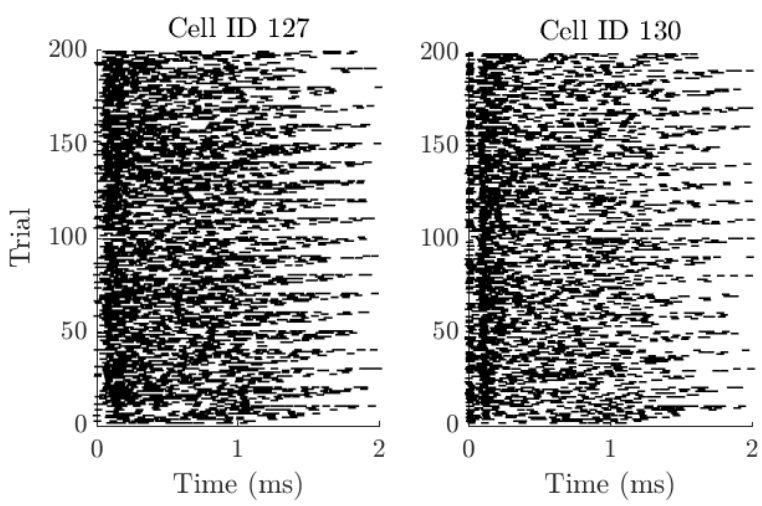

Figure 4. Raw event traces from neuron firing flicker experiment as described in Tang et al. [2015].
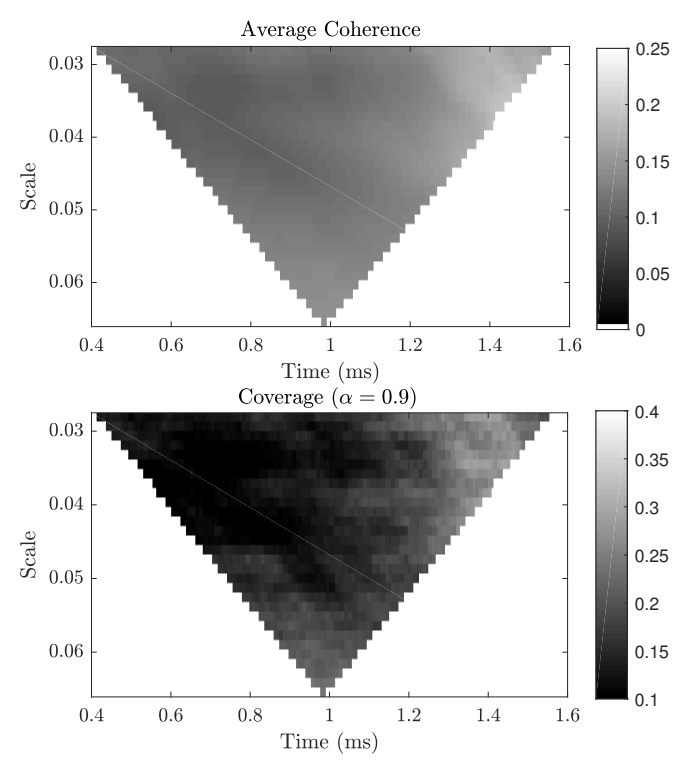

Figure 5. Top: Mean coherence, averaged across $N_{\text {trial }}=200$ recordings displayed in Fig. 4. Bottom: Proportion of estimates that are larger than the upper 90th percentile when $\kappa=12$.

Furthermore, each experiment is repeated a number of times, if we allow ourselves to generalise across experimental trials then we can compare the estimated coherence with what we would expect under a null hypothesis of zero dependence. Our findings are summarised in Fig. 5, where we plot the average coherence across all trials, and additionally, the number of estimates which exceed the $\alpha=0.1$ pointwise critical value derived from the Goodman distribution. Although, we only report the results here from the detrended series $\bar{X}[k]$ derived at binning level $\Delta=0.00782 \mathrm{~ms}$ (which gives $N=256$ ), we also assessed the coherence at a larger binning level, and using the raw (non-zero mean) process $X[k]$, the overall patterns observed in coherence were broadly consistent across these various settings.

Although we have only considered a single pair of neurons in this example, the results are still potentially quite interesting, and illustrate the utility of the wavelet methodology, i.e. to characterise how dependency may vary across time.
Clearly, we see some examples of this in the given example, where a coherent region is detected around $1.4 \mathrm{~ms}$ (at scale $a \approx 0.04)$ into the recording. Given the relatively low number of events in a single trial, in this example we report the number of coherence estimates $\hat{R}_{12}(a, b)$ which violate the 90th percentile of the null $(R=0)$ Goodman distribution, i.e. significance level $\alpha=0.1$. If there was zero coherence, we may expect this proportion to be around 0.1 , which appears consistent with the start of the recording period $(0.4-1 \mathrm{~ms})$. However, as we move to the latter stages of the recording, we see evidence for coherent signalling indicated by the fact that around $30-40 \%$ of the estimates in this region violate the 90th percentile of the null $(R=0)$ Goodman distribution.

\section{CONCLUSION}

In this paper, we have presented what we believe to be the first systematic study of the temporally smoothed wavelet coherence estimator applied to a discretised pointprocess. The aim of the work was to assess how appropriate the asymptotic distribution derived in the Gaussian, regular, time-series setting were to this kind of multivariate count time-series data. Our synthetic results verify that the temporally smoothed wavelet coherence can often be treated as being Goodman distributed under sampling. We verified, in the context of a Poisson process, that the rate parameter had little impact on the match of the empirical and asymptotic distributions. Further work is needed here to assess the behaviour of the estimator when correlation structure is present. For instance under self-exciting Hawkes, or Cox processes [Hawkes, 1971, Cox, 1955], or even locally stationary processes [Park et al., 2014, Gibberd and Nelson, 2016, Roueff et al., 2016].

In practice, we applied the estimator to a set of neuron firing data. An example of a complex, possibly dependent, non-stationary process. In this setting, we compared the estimated (temporally smoothed) coherence against the distribution expected under the null hypothesis of a stationary, independent process with zero true coherence. Even in the case where we have very few points, we demonstrated a method to assess evidence against the null by plotting the ratio of significant coherence estimates at the $\alpha$ level. This method indicates that while the neuron firing, in this case started off appearing incoherent, after some short time period $(\approx 1.4 \mathrm{~ms})$ coherent signalling may develop. Future work should look at a more systematic study of these developing correlation structures from a neuroscience perspective. We may also consider applying the temporally smoothed wavelet estimator directly to the event data itself, i.e. with no binning and comparing to alternative methods such as the recent smoothing proposal by Halliday et al. [2018].

\section{REFERENCES}

M. S. Bartlett. The Spectral Analysis of Point Processes. Journal of the Royal Statistical Society. Series B, 25(2): 264-296, 1963. 
J. Bigot, M. Longcamp, F. Dal Maso, and D. Amarantini. A new statistical test based on the wavelet cross-spectrum to detect time-frequency dependence between non-stationary signals: Application to the analysis of cortico-muscular interactions. NeuroImage, 55(4):1504-1518, 2011.

D. R. Brillinger. The spectral analysis of stationary interval functions. Proceedings of the Sixth Berkeley Symposium on Mathematical Statistics and Probability, Volume 1: Theory of Statistics, pages 483-513, 1972.

D. R. Brillinger. Nerve Cell Spike Train Data Analysis : A Progression of Technique. Journal of the American Statistical Association, 87(June), 1992.

D. R. Brillinger, H. L. Bryant Jr., and J. P. Segundo. Identification of Synaptic Interactions. Biol. Cybernetics, 228:213-228, 1976.

E. A. K. Cohen. Multi-wavelet coherence for point processes on the real line. ICASSP, IEEE International Conference on Acoustics, Speech and Signal Processing - Proceedings, (2):2649-2653, 2014.

E. A. K. Cohen and A. T. Walden. A Statistical Study of Temporally Smoothed Wavelet Coherence. IEEE Transactions on Signal Processing, 58(6):2964-2973, 2010.

D. R. Cox. Some Statistical Methods Connected with Series of Events. Journal of the Royal Statistical Society B, 17: 129-164, 1955.

A. J. Gibberd and J. D. B. Nelson. Estimating multiresolution dependency graphs within the stationary wavelet framework. In GlobalSIP, IEEE Global Conference on Signal and Information Processing, 2016.

N. R. Goodman. Statistical Analysis Based on a Certain Multivariate Complex Gaussian Distribution (An Introduction). The Annals of Mathematical Statistics, 34(1): 152-177, 1963.

D. M. Halliday and J. R. Rosenberg. Time and Frequency Domain Analysis of Spike Train and Time Series Data. 1999.

D. M. Halliday, J. S. Brittain, C. W. Stevenson, and R. Mason. Adaptive spectral tracking for coherence estimation: The z-tracker. Journal of Neural Engineering, 15(2), 2018.

A. G Hawkes. Point Spectra of Some Mutually Exiting Point Processes. Journal of the Royal Statistical Society, 33(3): 438-443, 1971.

A. E. Hramov, A. A. Koronovskii, V. A. Makarov, A. N. Pavlov, and E. Sitnikova. Wavelet Approach to the Study of Rhythmic Neuronal Activity, pages 177-209. Springer Berlin Heidelberg, 2015.

M. R. Jarvis and P. P. Mitra. Sampling Properties of the Spectrum and Coherency of Sequences of Action Potentials. Neural Computation, 749:717-749, 2001.

T. Park, I. A. Eckley, and H. C. Ombao. Estimating Time-Evolving Partial Coherence Between Signals via Multivariate Locally Stationary Wavelet Processes. IEEE Transactions on Signal Processing, 62(20):5240-5250, 2014.

F. Roueff, R. Von Sachs, and L. Sansonnet. Locally stationary Hawkes processes. Stochastic Processes and their Applications, 126(6):1710-1743, 2016.

J. Tang, S. C. Ardila Jimenez, S. Chakraborty, and S. R. Schultz. Visual receptive field properties of neurons in the mouse lateral geniculate nucleus. Plos One, 11(1): 1-34, 2015.

V. Ventura, C. Cai, and R. E. Kass. Statistical Assessment of Time-Varying Dependency Between Two Neurons. Journal of Neurophysiology, 94(4):2940-2947, 2005.

A. T. Walden. A unified view of multitaper multivariate spectral estimation. Biometrika, 87(4):767-788, 2000.

Y. Zhan, D. Halliday, P. Jiang, X. Liu, and J. Feng. Detecting time-dependent coherence between non-stationary electrophysiological signals-A combined statistical and timefrequency approach. Journal of Neuroscience Methods, 156(1-2):322-332, 2006. 